\title{
Komplementär- und Alternativmedizin in Europa
}

\author{
Zum Abschluss der Artikelserie zur ärztlichen Komplementärmedizin in der Schweiz \\ wirft dieser Beitrag den Blick über die Grenze nach Europa, wo die Komplementär- \\ und Alternativmedizin (CAM) eine ähnlich lang zurückreichende Tradition hat wie \\ in der Schweiz. Der Gebrauch der verschiedenen Methoden und Therapieverfahren \\ ist in der europäischen Bevölkerung ebenfalls weitverbreitet [1].
}

\author{
Klaus von Ammon ${ }^{a}$, \\ Barbara Kramer ${ }^{b}$, \\ Martin Frei-Erb ${ }^{a}$ \\ a Kollegiale Instanz für \\ Komplementärmedizin \\ KIKOM, Universität Bern \\ b UNION Schweizerischer \\ komplementärmedizinischer \\ Ärzteorganisationen
}

Korrespondenz:

Dr. med. Hansueli Albonico Praxis für Hausarzt- und Komplementärmedizin Bernstrasse 14 CH-3550 Langnau Tel. 0344021419

hu.albonico[at]hotmail.com

\section{Die Nachfrage nach Komplementärmedizin in Europa ist gross}

Schätzungen zeigen seit 1970, dass zwischen 10 und über 50\% der Bevölkerung Komplementärmedizin für Prophylaxe oder Erhalt und Wiederherstellung ihrer Gesundheit, insbesondere bei chronischen Krankheiten, nutzen [2]. Aufgrund der kulturellen Vielfalt Europas bestehen länderspezifische Unterschiede im Verständnis des Begriffes «Komplementär- und Alternativmedizin» und der damit in Zusammenhang stehenden Methoden und Heilmittel. Zudem existieren kaum wissenschaftliche Daten aus den einzelnen Ländern über die Häufigkeit des Gebrauchs der eingesetzten Therapieverfahren, über die Bedürfnisse und Einstellungen der Bevölkerung und über die gesetzlichen Vorschriften und Regelungen. Dies ist bedingt durch geringe Forschungsmöglichkeiten, da mit wenigen Ausnahmen kaum Forschungsgelder von öffentlicher und privater Hand oder von der pharmazeutischen Industrie zur Verfügung stehen. Gründe sind unter anderem, dass CAM in den europäischen Ländern häufig nicht im staatlich oder von obligatorischen Versicherungen finanzierten konventionellen Gesundheitsbereich ausgeübt wird und keine für die Industrie interessanten patentierbaren Behandlungsmöglichkeiten bietet. Private Initiativen vermögen diese Defizite nicht annähernd auszugleichen. Seit 10 bis 20 Jahren jedoch etabliert sich an Universitäten, vor allem in Deutschland, eine Forschungstradition in Form von Stiftungslehrstühlen. Um zur Klärung der offenen Fragen beizutragen, hat die Europäische Union EU im Rahmen des Forschungsprogramms FP7 das Projekt CAMbrella [3] (s. Kasten) finanziert.

\section{Vorschlag einer europäischen Definition von CAM}

Um gesamteuropäische Forschungsprojekte, Richtlinien und gesetzliche Vorschriften zu ermöglichen und zu vereinfachen, ist im Rahmen von CAMbrella folgender Vorschlag einer pragmatischen Definition von CAM entstanden:

«Komplementär- und Alternativmedizin (CAM), die in Europa genutzt wird, umfasst eine Vielfalt von medizinischen Systemen und Therapien. Deren Wis- sen, Fertigkeiten und Ausübung leiten sich von Theorien, philosophischen Systemen und praktischen Erfahrungen her, die sowohl für den Erhalt und die Verbesserung der Gesundheit als auch für Prophylaxe, Diagnose, Linderung, Behandlung oder Heilung körperlicher, psychischer oder geistiger Erkrankungen genutzt werden. CAM wurde bisher ausserhalb des konventionellen Gesundheitssystems genutzt, aber in manchen Ländern wurden bestimmte Behandlungsformen in die öffentliche Gesundheitsfürsorge übernommen oder an diese angepasst.» [4]

\section{Zuverlässige Informationen sind spärlich}

Zur Inanspruchnahme der Komplementärmedizin gibt es nur wenige hochwertige Untersuchungen in einzelnen Ländern (z. B. Deutschland, Grossbritannien, Schweiz). Die Prävalenz des Gebrauchs umfasst den Bereich von 0,3 bis $86 \%$, je nach Land, erfasstem Zeitraum und Krankheitsbild. Zusammenfassend macht die europäische Bevölkerung am meisten Gebrauch von Phytotherapie, nutzt die CAM überwiegend für muskulo-skelettale Probleme, und dies oft nach unbefriedigenden Ergebnissen der konventionellen Medizin. Kinder und Jugendliche, Patienten mit chronischen Erkrankungen und polymorbide Patienten sind die Bevölkerungsgruppen, die Komplementärmedizin am häufigsten in Anspruch nehmen [5].

Aus 18 von 27 EU-Staaten und 12 assoziierten Ländern gibt es Daten zu Bedarf und Erwartungen

\section{CAMbrella}

CAMbrella besteht aus einem Netzwerk von 16 akademischen Forschungsgruppen aus 12 europäischen Ländern. Aus der Schweiz sind die Universitäten Bern und Zürich vertreten. Im Zeitraum von 2010 bis 2012 haben neun Arbeitsgruppen eine Bestandsaufnahme zur Nachfrage, zum Angebot, zur Gesetzeslage und zur Regulierung sowie zu den Bedürfnissen und Einstellungen der Bevölkerung in 39 europäischen Ländern durchgeführt. Als Resultat ist eine zukünftige europäische Forschungsstrategie betreffend CAM entwickelt und in Brüssel Mitgliedern des EU-Parlaments und der Öffentlichkeit vorgestellt worden. 


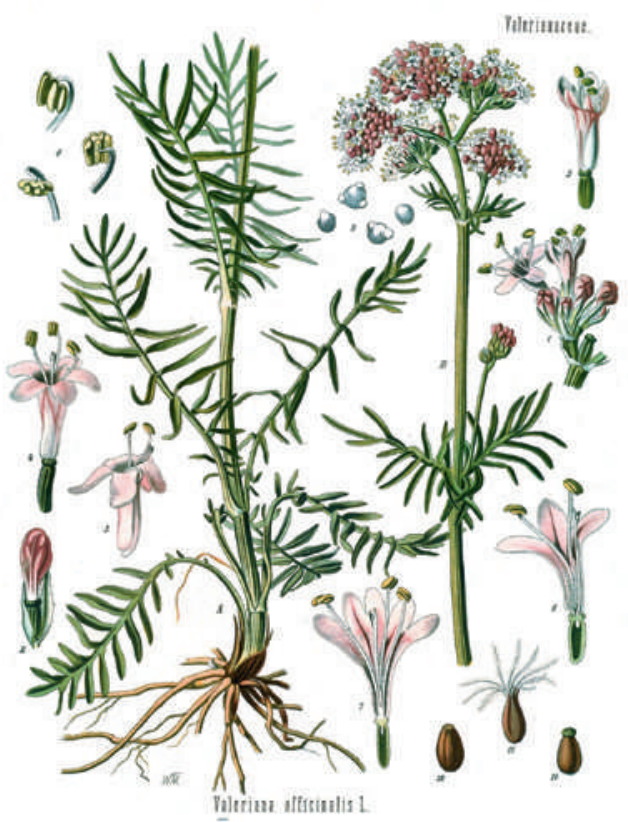

Der Echte Arznei-Baldrian (Valeriana officinalis) hat eine lange Tradition als Arzneimittel in der Pflanzenheilkunde.

der Bevölkerung hinsichtlich Komplementärmedizin, und nur für Grossbritannien und die Schweiz sind differenzierte Ergebnisse vorhanden. Mit diesen Einschränkungen lassen sich folgende Tendenzen feststellen: Die europäische Bevölkerung wünscht allgemein erhältliche spezifische Information, persönliche Beratung und weitgehend ungehinderten Zugang zu verschiedenen komplementärmedizinischen Angeboten in Praxen und Spitälern. Dies soll nicht nur durch ärztliches Personal erfolgen, sondern auch durch spezifisch ausgebildete therapeutisch Tätige, deren Leistungen möglichst im Rahmen des öffentlichen Gesundheitswesens bezahlt werden sollen [6]. Dies steht im Gegensatz zur gegenwärtigen Haltung vieler konventionell Tätigen und der aktuellen Praxis der privaten «Out-of-pocket»- oder Zusatzversicherungs-Aufwendungen.

Gegenwärtig bieten in Europa (rund 500 Millionen Einwohner) ca. 150000 Ärzte mit einer Zusatzqualifikation in CAM und ca. 180000 registrierte und zertifizierte nicht-ärztliche Therapeut(inn)en komplementärmedizinische Methoden und Techniken an [7]. Auf 100000 Einwohner bezogen sind das 35 CAM-Therapeuten und 30 CAM-Ärzte im Vergleich zu 95 konventionellen Allgemeinärzten. Akupunktur wird am häufigsten angeboten: Mit 80000 Ärzten und 16000 Therapeuten sind es 30\% aller Anbietenden. Homöopathie (45000 Ärzte/5800 Therapeuten), Phytotherapie (29000 Therapeuten) und 25000 Reflex-Therapeuten stellen das zweite Drittel. Alle anderen Therapien zusammen bestreiten das dritte Drittel des Angebots. Davon werden nur Naturheilverfahren (meist in Deutschland) mit 22500, Anthroposophische Medizin (4500) und Neuraltherapie (1500) vorwiegend von Ärzten ausgeübt.
Darüber hinaus praktizieren in Europa Personen Komplementär- und Alternativmedizin innerhalb des gesetzlichen Rahmens, die es vorziehen, sich nicht für spezifische Methoden registrieren oder zertifizieren zu lassen (z. B. «Energetiker» in Österreich). Diese Anbieter lassen sich kaum systematisch erfassen und vergleichen. Die Dunkelziffer beträgt z. B. für Osteopathie in Italien mehr als 50\% [8]. Für die meisten dieser Verfahren gibt es nationale und europäische Dachorganisationen, teilweise mit einheitlichen, zum Teil verbindlichen Qualitätsstandards in Aus-, Weiter-, Fortbildung und ethischen Richtlinien.

Die Zulassungsbedingungen und Vorschriften betreffend Zertifizierung und Fortbildung sind in jedem europäischen Land unterschiedlich geregelt, ähnlich wie wir dies von den 26 Kantonen in der Schweiz kennen: grosse Freizügigkeit (z.B. Naturärzte und -ärztinnen in Appenzell Ausserrhoden) bis zu gesamtstaatlichen Regelungen (z.B. Fähigkeitsausweise der FMH). Eine allgemeine Gesetzgebung gibt es in 19 von 39 Ländern, von denen 11 spezifische Gesetze zur Komplementär- und Alternativmedizin haben und acht weitere Länder Komplementär- und Alternativmedizin in ihre Gesundheitsgesetzgebung eingeschlossen haben [9].

Wie bei der Gesetzgebung spielt die Schweiz auch bei der Harmonisierung der Zulassungsbestimmungen eine Vorreiterrolle in Europa: Im Zusammenhang mit dem 2009 vom Stimmvolk angenommen Verfassungsartikel zur Komplementärmedizin sind eidgenössisch anerkannte Diplome für Naturheilpraktiker und komplementärmedizinische Therapeuten in Arbeit. Die öffentliche Anerkennung, die Qualitätssicherung, ethische Richtlinien für Therapeuten und ein gleichberechtigter Zugang zu CAM erhöhen das Vertrauen der Bevölkerung in CAM. Eine einheitliche Handhabung auf europäischem Niveau würde der Bevölkerung eine Qualitätssicherheit bei der Inanspruchnahme komplementärmedizinischer Therapien ermöglichen.

\section{Qualifizierte ärztliche Komplementärmedizin ist wirtschaftlich}

In der Schweiz hat das staatlich finanzierte Programm Evaluation Komplementärmedizin (PEK) für fünf Bereiche qualifizierter ärztlicher Komplementärmedizin je nach Methode vergleichbare, leicht oder deutlich verminderte Kosten im Vergleich zur konventionellen Allgemeinmedizin-Versorgung dokumentiert [10-12]*. Eine derartig differenzierte Untersuchung fehlt bisher für andere europäische Länder. Eine vergleichbare Studie gibt es einzig aus den Niederlanden, die zu einem ähnlichen Ergebnis kommt [13]. Für einzelne Verfahren bei bestimmten Beschwerden oder Indikationen gibt es darüber hinaus qualitativ hochwertige Untersuchungen, die («non-inferiority») Kosteneffizienz oder auch Kostenvorteile nachweisen, wie eine aktuelle systematische Übersichtsarbeit belegt [14]. Viele CAM-Be- 
handlungen sind Beratungen oder benötigen keine, geringe oder kostengünstige Hilfsmittel und stellen daher keinen bedeutenden Industrie-Faktor dar. Für ärztliche Komplementärmedizin werden in der Schweiz pro Jahr ca. 250 Millionen Franken aufgewendet. Das entspricht knapp 0,4\% der jährlichen Gesundheitskosten 2011 von 64,6 Milliarden Franken [15]. In der gleichen Grössenordnung bewegen sich die Zahlen des konventionellen und komplementär-alternativen Pharmamarkts in Europa. Hersteller von CAM-Produkten sind meist kleine und mittlere Unternehmen, das Hauptabsatzgebiet sind phytotherapeutische und homöopathische Produkte sowie Nahrungsergänzungsmittel. In Europa werden vorwiegend pflanzliche Produkte umgesetzt, was 2011 etwa 6 Milliarden Euro entspricht. Der Exfactory-Preis homöopathischer Produkte summiert sich $2010 \mathrm{zu}$ ca. 1 Milliarde Euro, was 0,7\% des Pharma-Markts in Europa entspricht [16].

\section{«Es wäre wünschenswert, die staatliche Forschungs-}

\section{Unterstützung zu erhöhen.»}

\section{Zusammenfassung und Ausblick}

Die anhaltend hohe Nachfrage der Bevölkerung nach CAM ist europaweit nachgewiesen. Im Vergleich mit Europa wird deutlich, wie gut in der Schweiz vor allem die ärztliche, aber auch die nichtärztliche Komplementär- und Alternativmedizin strukturell organisiert und funktionell wie finanziell untersucht sind. Dank der Möglichkeit einer direktdemokratischen politischen Einflussnahme nimmt die Schweiz eine Sonderstellung innerhalb von Europa ein. In Anbetracht der grossen Nachfrage seitens der Patienten, der offenen Fragen und der mangelnden Unterstützung durch private Firmen wäre es wünschenswert, die staatliche Forschungs-Unterstützung zu erhöhen. So fordern auch die Forscher des CAMbrella-Projekts die EU auf, europäische CAM-Forschungsprogramme zu implementieren: «Wenn CAM ein Teil der Lösung der Probleme im Gesundheitswesen sein soll, die in den kommenden Jahren auf uns zukommen, müssen wir dringend zuverlässige Informationen über Wirksamkeit, Sicherheit und Kosten in den realen Versorgungsbedingungen sammeln und analysieren.» [17]

\section{Literatur}

1 Ong CG, Bodeker G, Grundy C, et al. WHO Global Atlas of Traditional, Complementary and Alternative Medicine. Map Volume. WHO Centre for Health Development. Kobe. Japan; 2005.

2 Maddalena S. Alternative Medicines: On the Way towards Integration? Chapter 2.2. Bern: Peter Lang; 2005.
3 www.cambrella.eu

4 Falkenberg T, Lewith G, Roberti di Sarsina P, et al. Towards a Pan-European Definition of Complementary and Alternative Medicine - a Realistic Ambition? Forschende Komplementärmedizin / Research in Complementary Medicine. 2012;19(Suppl 2):6-8.

5 Eardley S, Bishop FL, Prescott P, et al. A Systematic Literature Review of Complementary and Alternative Medicine Prevalence in EU. Forschende Komplementärmedizin / Research in Complementary Medicine. 2012;19(s2):18-28.

6 Nissen N, Schunder-Tatzber S, Weidenhammer W, Johannessen H. What Attitudes and Needs Do Citizens in Europe Have in Relation to Complementary and Alternative Medicine? Forschende Komplementärmedizin / Research in Complementary Medicine. 2012;19(s2):9-17.

7 von Ammon K, Frei-Erb M, Cardini F, et al. Complementary and Alternative Medicine Provision in Europe - First Results Approaching Reality in an Unclear Field of Practices. Forschende Komplementärmedizin / Research in Complementary Medicine. 2012;19(Suppl 2):37-43.

8 Petracca M. Osteopathic medicine in Italy: a descriptive study. 9th International Congress on Advances in Osteopathic Research ICAOR. London; 2012. Erhältlich unter: www.bcom.ac.uk/downloads/A4_ ICAOR_9_Brochure_FFF_PDF.pdf (letztmals aufgerufen: 12.9.2013).

9 Wiesener S, Falkenberg T, Hegyi G, Hök J, Roberti di Sarsina P, Fønnebø V. Legal status and regulation of complementary and alternative medicine in Europe. Forsch Komplementmed. 2012;19 (Suppl 2):29-36.

10 Bundesamt für Gesundheit BAG. Schlussbericht PEK. 2005. Erhältlich unter: www.bag.admin.ch/themen/ krankenversicherung/00263/00264/04102/index. html?lang=de (letztmals aufgerufen: 12.9.2013).

11 Studer HP, Busato A. Ist ärztliche Komplementärmedizin wirtschaftlich? Schweiz Ärztezeitung. 2010;91(18):707-11.

12 Studer HP, Busato A. Comparison of swiss basic health insurance costs of complementary and conventional medicine. Forschende Komplementärmedizin (2006). 2011;18(6):315-20.

13 Kooreman P, Baars EW. Patients whose GP knows complementary medicine tend to have lower costs and live longer. Eur J Health Econ. 2012;13(6):769-76.

14 Herman PM, Poindexter BL, Witt CM, et al. Are complementary therapies and integrative care cost-effective? A systematic review of economic evaluations. BMJ Open. 2012;2:e001046. doi:10.1136/ bmjopen-2012-001046

15 Eidgenössisches Departement des Innern. Bundesamt für Statistik. Medienmitteilung vom 23.4.2013. Erhältlich unter: www.bfs.admin.ch/bfs/portal/de/ index/themen/14/01/new/nip_detail. html?gnpID=2013-209 (letztmals aufgerufen: 12.9.2013).

16 ECHAMP Homeopathy and anthroposophic medicine in the EU: Facts and Figures 2011 (Third edition), Erhältlich unter: www.echamp.eu/publications/ brochures.html (letztmals aufgerufen: 12.9.2013).

17 Brinkhaus B. Roadmap for European CAM research. Erhältlich unter: www.cambrella.eu/home. php?il=204\&I=deu (letztmals aufgerufen: 12.9.2013) 